\title{
AN INTERSECTION PROPERTY IN LOCALLY CONVEX SPACES
}

\author{
R. HARROP AND J. D. WESTON
}

Among the uniform spaces, those that are complete are characterized by the property that every family of closed sets which is directed by inclusion (forming a filter-base) and has arbitrarily small members, has a common point. If we omit the condition on the sizes of the sets we obtain a characteristic property of compact spaces. Nevertheless, there are certain spaces in which closed sets of a particular form always have this intersection property, irrespective of size, although the sets may not be compact. For example, it is known [1] that in a Banach space the closed sets that are bounded and convex have the property if and only if the space is reflexive. It is easy to show that the closed spheres in any Banach space have the property, although an example is known [2] of a complete metric space in which the closed spheres do not have the property. We show here that in a locally convex space, the sets obtained by translating and magnifying a given sequentially closed bounded set have the intersection property if the closed symmetric convex hull of the given set is sequentially complete. (This, of course, includes the case of the closed spheres in a Banach space.)

We assume throughout that $X$ is a locally convex linear Hausdorff space, and that $B$ is any fixed set in $X$ which is bounded and sequentially closed and has more than one point.

LEMmA 1. Suppose that $x_{1}+\rho_{1} B \subseteq x_{2}+\rho_{2} B$, where $x_{1}, x_{2} \in X$ and $\rho_{1}, \rho_{2}$ are non-negative numbers. Then $\rho_{1} \leqq \rho_{2}$ and $x_{1}-x_{2} \in\left(\rho_{2}-\rho_{1}\right) B$.

PRoof. A sequence $\left\{y_{n}\right\}$ in $B$ may be constructed as follows: taking $y_{0}$ to be any point of $B$, assume that, for some $n>0, y_{n-1}$ has been defined; then $B$ has a point $y_{n}$ such that

$$
x_{1}+\rho_{1} y_{n-1}=x_{2}+\rho_{2} y_{n} .
$$

If $\rho_{1}=\rho_{2}=\rho$, this gives

$$
\rho\left(y_{n}-y_{0}\right)=n\left(x_{1}-x_{2}\right),
$$

so that $x_{1}-x_{2} \in(\rho / n)\left(B-y_{0}\right)$ for all $n$. Since $B-y_{0}$ is bounded, it follows that, in this case,

Received by the editors February 28, 1955 and, in revised form, May 25, 1955. 


$$
x_{1}-x_{2}=0 \in\left(\rho_{2}-\rho_{1}\right) B .
$$

If $\rho_{1} \neq \rho_{2}$ then $\rho_{2} \neq 0$ (since $B$ has more than one point) and we have, by induction,

$$
y_{n}=\left(\rho_{1} / \rho_{2}\right)^{n} y_{0}+\frac{1-\left(\rho_{1} / \rho_{2}\right)^{n}}{\rho_{2}-\rho_{1}}\left(x_{1}-x_{2}\right),
$$

that is,

$$
y_{n}-x_{0}=\left(\rho_{1} / \rho_{2}\right)^{n}\left(y_{0}-x_{0}\right),
$$

where $x_{0}=\left(\rho_{2}-\rho_{1}\right)^{-1}\left(x_{1}-x_{2}\right)$. We can suppose that $y_{0} \neq x_{0}$. Then, since $y_{n}-x_{0}$ belongs to the bounded set $B-x_{0}$, we must have $\rho_{1} / \rho_{2}<1$, that is, $\rho_{1}<\rho_{2}$. It then follows that $y_{n} \rightarrow x_{0}$ as $n \rightarrow \infty$, so that $x_{0} \in B$ (since $B$ is sequentially closed). Thus $x_{1}-x_{2} \in\left(\rho_{2}-\rho_{1}\right) B$.

Lemma 2.' Suppose that the closed symmetric convex hull of $B$ is sequentially complete, and let $x_{0}$ be any point of $X$. Let $L$ be the subspace generated by the closed symmetric convex hull of $B \cup\left(x_{0}\right)$. Then $L$ is a Banach space with respect to a topology stronger than the induced topology.

Proof. Let $S_{1}$ and $S_{2}$ be the closed symmetric convex hulls of $B$ and of $B \cup\left(x_{0}\right)$ respectively. Let $A$ be the set $\left\{\alpha x_{0}:|\alpha| \leqq 1\right\}$. The set $S_{1}+A$ is obviously symmetric and convex; it is also closed, since $A$ is compact. (For, suppose that $x \in S_{1}+A$. Then if $a \in A, x$ is outside the closed set $S_{1}+a$; hence a neighborhood $U_{a}$ of 0 exists such that $U_{a}-U_{a}+x$ does not meet $S_{1}+a$, that is, $U_{a}+x$ does not meet $S_{1}+U_{a}+a$. For some finite set $a_{1}, \cdots, a_{n}, A \subseteq \bigcup_{i=1}^{n}\left(U_{a_{i}}+a_{i}\right)$; then $\bigcap_{i=1}^{n} U_{a_{i}}+x$ is a neighborhood of $x$ which does not meet $S_{1}+A$.) Thus $S_{2} \subseteq S_{1}+A$, so that if $\left\{x_{n}\right\}$ is any sequence in $S_{2}$ we have $x_{n}=y_{n}+a_{n}$, where $y_{n} \in S_{1}$ and $a_{n} \in A$. From the sequential completeness of $S_{1}$ and the compactness of $A$, it follows that if $\left\{x_{n}\right\}$ is a Cauchy sequence it is convergent. $S_{2}$ is therefore sequentially complete.

For any $x \in L$, let $\|x\|=\inf _{\rho>0, \rho x \in S_{2}}(1 / \rho)$. This defines a norm for $L$, with respect to which $S_{2}$ is the unit ball $(\|x\| \leqq 1)$. The topology given by this norm is stronger than the induced topology, since, if $U$ is any closed convex neighborhood of 0 in $X$ then, for some $\delta>0, \delta\left(B \cup\left(x_{0}\right)\right) \subseteq U$ and hence $\delta S_{2} \subseteq U$. Thus if $\left\{x_{n}\right\}$ is a Cauchy sequence in $L$ with respect to the norm, it is a Cauchy sequence with respect to the induced topology. For any $\epsilon>0$, let $n_{0}$ be an integer such that, when $m, n>n_{0},\left\|x_{m}-x_{n}\right\|<\epsilon$, that is,

1 This is essentially an adaptation to our purpose of a result of Bourbaki [3]. 


$$
x_{n} \in \epsilon S_{2}+x_{m} \text {. }
$$

Then, since $\epsilon S_{2}$ is sequentially complete, $\left\{x_{n}\right\}$ has a limit $x^{*}$; and, since $\epsilon S_{2}$ is closed, we have, for all $m>n_{0}, x^{*} \in \epsilon S_{2}+x_{m}$, that is, $\left\|x_{m}-x^{*}\right\|<\epsilon$. Thus $\left\{x_{n}\right\}$ is convergent with respect to the norm, so that $L$ is a Banach space.

In stating our main result, we use the symbol " $\Lambda$ " to denote some partially ordered set which contains, with any two of its elements $\lambda, \mu$, an element $\nu$ such that $\nu \geqq \lambda$ and $\nu \geqq \mu$. By a directed system of sets in a given space, we mean a family $\left\{E_{\lambda}\right\}_{\lambda \in \Lambda}$, indexed by a $\Lambda$, such that $E_{\lambda} \subseteq E_{\mu}$ if and only if $\lambda \geqq \mu$. If $\left\{x_{\lambda}\right\}_{\lambda \in \Lambda}$ is a family of points indexed by a $\Lambda$, the sets of the form $\left\{x_{\lambda}: \lambda \geqq \lambda_{0}\right\}$, where $\lambda_{0}$ runs through $\Lambda$, constitute a directed system; if the filter generated by this has a unique limit, we denote this limit by "lim $x_{\lambda}$." (In a complete uniform space the limit exists if and only if the filter has arbitrarily small members.)

Theorem. Suppose that the closed symmetric convex hull of $B$ is sequentially complete (this hypothesis is satisfied in particular if $X$ is complete). Let $\left\{B_{\lambda}\right\}_{\lambda \in \Lambda}$ be a directed system of sets of the form $B_{\lambda}$ $=x_{\lambda}+\rho_{\lambda} B$, where $\rho_{\lambda} \geqq 0$. Then $\bigcap_{\lambda \in \Lambda} B_{\lambda}$ is a set $x+\rho B$, with $x=\lim x_{\lambda}$ and $\rho=\inf _{\lambda \in \Lambda} \rho_{\lambda}=\lim \rho_{\lambda}$.

Proof. We may assume that all the sets $B_{\lambda}$ are contained in some fixed set $x_{0}+\rho_{0} B$ (corresponding to some particular value of $\lambda$ ), with $\rho_{0}>0$. These sets are then contained in the Banach space $L$ of Lemma 2, and are closed and bounded with respect to the norm.

Let $\epsilon$ be any positive number. If $\rho=\inf _{\lambda \in \Lambda} \rho_{\lambda}$, we can choose $\lambda_{0} \in \Lambda$ so that $\rho \leqq \rho_{\lambda_{0}}<\rho+\epsilon$. Suppose that $\lambda \geqq \lambda_{0}$ and $\mu \geqq \lambda_{0}$. Then $B_{\lambda} \subseteq B_{\lambda_{0}}$ and $B_{\mu} \subseteq B_{\lambda_{0}}$ so that, by Lemma $1, \rho \leqq \rho_{\lambda} \leqq \rho_{\lambda_{0}}$ and $\rho \leqq \rho_{\mu} \leqq \rho_{\lambda_{0}}$; thus, since $\epsilon$ is arbitrary, $\rho=\lim \rho_{\lambda}$. Lemma 1 also gives

$$
x_{\lambda}-x_{\lambda_{0}} \in\left(\rho_{\lambda_{0}}-\rho_{\lambda}\right) B \text { and } x_{\mu}-x_{\lambda_{0}} \in\left(\rho_{\lambda_{0}}-\rho_{\mu}\right) B \text {. }
$$

Now $B$, being bounded, lies in some sphere $\|x\|<\delta$; thus $\left\|x_{\lambda}-x_{\mu}\right\|$ $<2 \epsilon \delta$ when $\lambda \geqq \lambda_{0}$ and $\mu \geqq \lambda_{0}$. Since $L$ is complete, it follows that $\lim x_{\lambda}$ exists (with respect to the norm, and hence with respect to the topology of $X)$. Let $x=\lim x_{\lambda}$.

Now let $\lambda \in \Lambda$ and $y \in B$. There is a $\mu \in \Lambda$ such that

$$
\left\|x_{\nu}+\rho_{\nu} y-x-\rho y\right\|<\epsilon \text { if } \nu \geqq \mu,
$$

and we can choose $\nu$ so that $\nu \geqq \lambda$ and $\nu \geqq \mu$. Thus $B_{\lambda}$ has points, $x_{\nu}+\rho_{\nu} y$, arbitrarily close to $x+\rho y$. Since $B_{\lambda}$ is closed, it follows that $x+\rho B \subseteq B_{\lambda}$. Thus $x+\rho B \subseteq \bigcap_{\lambda \in \Lambda} B_{\lambda}$. On the other hand, suppose that $z \in \bigcap_{\lambda \in \Lambda} B_{\lambda}$. Then, for every $\lambda \in \Lambda$, we have $z=x_{\lambda}+\rho_{\lambda} y_{\lambda}$, where $y_{\lambda} \in B$. 
If $\rho=0$ then, since $B$ is bounded, $z=x$, so that $z \in x+\rho B$. If $\rho \neq 0$ then $y_{\lambda}=\left(1 / \rho_{\lambda}\right)\left(z-x_{\lambda}\right)$, so that $\lim y_{\lambda}$ exists and is $(1 / \rho)(z-x)$ : thus $z=x+\rho \lim y_{\lambda}$, so that, since $B$ is closed, $z \in x+\rho B$. We have now proved that $\bigcap_{\lambda \in \Lambda} B_{\lambda}=x+\rho B$.

We wish to acknowledge a referee's suggestion which enabled us to sharpen our original result.

\section{REFERENCES}

1. V. Smulian, On the principle of inclusion in the space of type (B), MatematiðeskiY Sbornik vol. 5 (47) (1939) pp. 317-328.

2. W. Sierpinski, Sur une définition des espaces complets, Ganita vol. 1 (1950) pp. 13-16.

3. N. Bourbaki, Sur certains espaces vectoriels topologiques, Ann. Inst. Fourier Grenoble vol. 2 (1950) pp. 5-16.

UNIVERSITY OF DURHAM 